ciliary agglutination ciliary dyskinesia

$\lg M$ mytilus edulis

serum immunoglobulins

\title{
Serum Proteins Agglutinate Cilia and Modify Ciliary Coordination
}

\author{
M. J. SANDERSON ${ }^{(5,4)}$ AND M. A. SLEIGH \\ Department of Biology. University' of Southampton. Southampton, United Kingdom
}

\begin{abstract}
Summary
A quantifiable assay is described in which the lateral cilia of the sea mussel Mytilus edulis are used to examine the effects of serum on ciliary function.

Human sera and sera from a variety of animals have been found to induce a change in ciliary metachrony. This alteration in activity appears to be brought about by the agglutination of adjacent cilia into blocks, with the effect of increasing the wavelength; the beat frequency remains steady. Parallel studies with the rabbit cilia assay have shown the agglutination of tracheal cilia into clumps by adult bovine serum. The dilution of adult bovine serum lengthens the time before dyskinesia is first observed in both the $M y$ tilus and rabbit assays. Fetal bovine or human cord sera do not cause ciliary dyskinesia or ciliary agglutination.

The evidence presented shows that the observed ciliary dyskinesia can be induced by sera from a variety of animals and is not specific to cystic fibrosis; it also suggests that the serum immunoglobulin fraction is responsible for these effects-initial studies implicate IgM.
\end{abstract}

\section{Speculation}

The discrimination between whole sera from normal and cystic fibrosis subjects by some other workers using ciliary assays may depend upon differences in the quantity of ciliary-specific serum immunoglobulins, presumably IgM, between the normal and cystic fibrosis serum samples. The effect of any small molecular weight factor found after fractionation in the other studies must be completely overshadowed in whole sera by the effect of IgM.

Cystic fibrosis (CF) is a lethal genetic condition of caucasian children which is inherited in an autosomal recessive manner. The disease seriously affects the exocrine glands, with the lungs being severely impaired by the accumulation of tenacious mucus $(29-30,31,43,56)$.

In 1967, Spock et al. (51) reported that sera from CF children and their parents (the heterozygotes or carriers of the disease), induced an "asynchronous beat" of rabbit tracheal cilia; healthy donors had no such effect. This finding was of great interest, and similar work was initiated in several laboratories using a variety of ciliated tissues. The results of these studies have suggested the presence of a circulating ciliostatic factor, which may reflect the basic gene defect, with the possible prospect of prenatal diagnosis; however, many of these findings have been the subject of some controversy. Bowman et al. $(13,15,16)$ and Lockhart and Bowman (42) have reported that CF sera cause the cessation of activity of the cilia of oyster gills within $30 \mathrm{~min}$; ciliary activity persisted for 40 to $50 \mathrm{~min}$ with sera from normal donors or patients with nonCF pulmonary disease. A similar assay using the cilia of the freshwater mussel Dreissensia polymorpha has been found to demonstrate earlier ciliary cessation due to CF serum $(10,26)$. Conover et al. (21) detected ciliary dyskinesia (a disturbance of the normal rhythm of activity) within 3 to $6 \mathrm{~min}$ with CF sera using a modified rabbit tracheal assay of Spock et al. (51); with normal sera dyskinesia only occurred after longer exposure.

These assays have been used extensively in an attempt to isolate and identify a "CF factor" responsible for dyskinesia. Evidence has been found suggesting this factor to be proteinaceous, heat labile $(4,14,51)$, and with a molecular weight between 5,000 and 10,000 daltons $(5,14,20)$. It is believed not to be an immunoglobulin (37), although it is often closely, but not covalently, associated with $\operatorname{IgG}(6,14,20,28)$. The success in demonstrating the presence of a serum factor is challenged by several other studies using freshwater mussels $(18,48)$, oysters, rabbit tracheal cilia (18), and sea mussel cilia (10) in which CF sera could not be distinguished from normal sera. Cherry et al. (17) have reported that all sera types are ciliotoxic. Other ciliated organisms such a protozoa and sperm could not be used successfully to discriminate between $C F$ and normal sera (44).

This disagreement between assays and the range of results obtained within a positive assay cast serious doubt on the specificity and usefulness of this phenomenon. It has been shown that ciliary dyskinesia similar to that induced by $C F$ sera can be induced by the sera of other diseases, e.g., bronchial asthma (23).

A possible explanation for the range of results obtained may lie within the tissue itself and in the methods of ciliary assessment. For an assay system to be of value, it must possess a number of characteristics, namely, simple and repeatable tissue preparation, clear ciliary observation, and a quantitative measurement of ciliary activity and metachronism. All of the assays used to date fail in one or more of these requirements and, although they may be of initial use in determining the presence of a factor, little else can be learned from the results with respect to understanding the dyskinesia.

Rabbit tracheal cilia are small and densely packed on an opaque mucosa, which makes their observation extremely difficult. Recent work in our laboratory has shown that normal ciliary activity on cultured tracheal epithelia is complicated and without a clear metachronism (49). These inherent problems of studying rabbit cilia have been recognized by Verdugo et al. (54), who have attempted to quantify the rabbit assay using laser beam scattering to measure beat frequency. This method, as they have indicated. lacks the ability to determine the characteristics and extent of the coordination between the cilia, so direct comparisons of the results with previous assays cannot be made. Similar problems of observation and quantification exist with the oyster gill cilia assay; these shortcomings are demonstrated by the absence of any clear description of the characteristics of ciliary dyskinesia.

Clearly, there is a need for an assay system which can overcome these problems. This study describes a bioassay using the lateral cilia of the sea mussel Mytilus edulis. These cilia are clearly visible and beat regularly with a prominent metachrony, any changes in beat frequency or metachronal coordination being immediately obvious. The ciliary activity can be easily quantified by a slit camera technique (52) so that a number of parameters, and not 
just the time before the appearance of an abnormal beat, can be measured. Finally, sea mussel cilia have been the subject of many productive investigations and their normal activity is quite well understood $(1,2,8,34,35,39)$.

The nature of the ciliary dyskinesia caused by sera from a variety of human and animal sera has been investigated using this Mytilus assay system. A comparison of the results with the effects of these sera on rabbit tracheal cilia has also been made.

\section{MATERIALS AND METHODS}

The effects of ciliary activity were examined by two bioassays, one using the lateral cilia of the sea mussel Mytilus edulis $(8,39)$ and the other using the tracheal cilia of rabbits.

\section{THE MYTILUS ASSAY}

Sea mussels were collected from Southampton Water at regular intervals and were maintained in a marine aquarium until required. The animal was opened by cutting the anterior and posterior adductor muscles to separate the shells and the gill lamellae were then dissected from the viscera. The complete gill was transferred to fresh natural seawater where individual filaments were separated from the inner gill lamella. These were washed in natural seawater.

For the bioassay, an individual filament was mounted in a perfusion chamber with the frontal cilia uppermost, each end of the filament being held between two glass chocks attached to a glass slide by a thin layer of silicone grease (Fig. 1). The fluid in the chamber could be replaced by the simultaneous movement of the inlet and outlet syringe plungers. The chamber was cooled to a temperature of 12 to $14^{\circ} \mathrm{C}$ by a water-jacketed microscope stage. Only those filaments which were in good condition, initially indicated by active latero-frontal cilia, followed by a uniform response to 5-hydroxytryptamine (5-HT), were used.

Each filament was perfused with artificial sea water (ASW) (35) (423 mM NaCl, $9 \mathrm{mM} \mathrm{KCl}, 9.27 \mathrm{mM} \mathrm{CaCl}_{2}, 22.9 \mathrm{mM} \mathrm{MgCl}$,

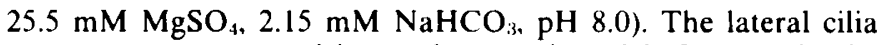
come to rest upon excision and are activated before use in the bioassay by a second perfusion with ASW containing $10^{-5}$ M 5HT (34). Serum or serum proteins, which had previously been dialyzed against ASW, were diluted to $50 \%$ with ASW and 5-HT was added to final concentration of $10^{-5} \mathrm{M}$. A period of $15 \mathrm{~min}$ was allowed for metachronal stabilization before the test solution was perfused. Any effects were observed and recorded by light microscopy as described below.

\section{THE RABBIT CILIA ASSAY}

The tracheal cilia of New Zealand White rabbits were used exclusively. The rabbits were killed by the injection of Nembutal $(0.6 \mathrm{mg} / \mathrm{ml}, 1 \mathrm{ml} / \mathrm{kg}$ body weight $)$, into the ear vein. The trachea was aseptically removed and transferred to Eagle's minimal essential medium (MEM) (modified), with Hanks' salts and $20 \mathrm{mM} \mathrm{4-}$ (2-hydroxyethyl)-1-piperazineethanesulfonic acid buffer (Flow Laboratories, Irvine, Scotland). The trachea was opened and pinned out so that the mucosa could be dissected off. Small pieces of mucosa were placed in organ culture on stainless steel grids with MEM, 10\% fetal bovine serum (FBS), penicillin ( $100 \mathrm{IU} / \mathrm{ml}$ ), and streptomycin $(100 \mu \mathrm{g} / \mathrm{ml})$ (Flow Laboratories) and incubated at $38^{\circ} \mathrm{C}$ until required. The medium was changed at 48 hourly intervals.

For the assay, the tissue was washed in MEM and cut into thin slices; several of these were placed on their sides on a slide so that the ciliary borders were clearly visible in profile view, care being taken to prevent the crushing of the tissue. The ciliary activity could then be examined before and after exposure to serum solutions. Test solutions, (sera diluted with phosphate-buffered saline), were drawn under the coverslip by placing a piece of filter paper at the opposite edge of the coverslip.

\section{ISOLATION OF MYTILUS CILIA}

The method used for ciliary isolation has been adopted after several trials with combinations of existing methods. It was found

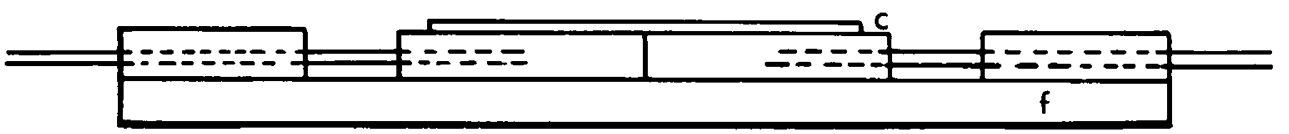

(i)

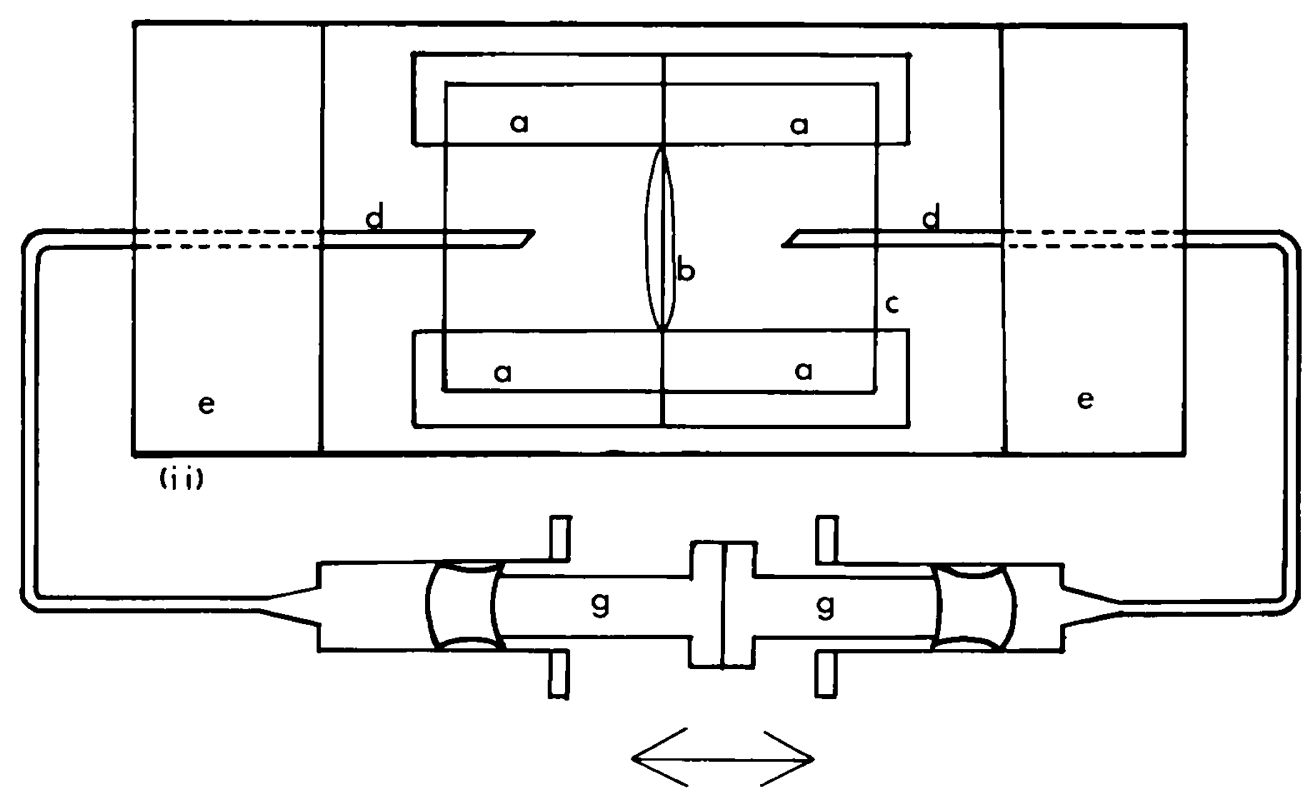

Fig. 1. Diagrams of the perfusion chamber and fluid exchange mechanism used for Mytilus assay. A side view (i) and plan view (ii) show two pairs of glass chocks $(a)$, attached to a glass slide $(f)$ by a thin layer of silicone grease, holding each end of the $M_{y}$ tilus gill filament $(b)$. The preparation is covered by a coverslip $(c)$ under which are positioned the inlet and outlet pipes $(d)$ secured by two mounts $(e)$. Fluid is exchanged by the simultaneous movement of the syringe plungers $(g)$. 
that hypertonic seawater (41) produced excessive mucus which trapped many of the isolated cilia, thus reducing yield. The ethanol/calcium method (33) was not used because it may damage the ciliary membranes. The method followed was simply the immersion of the gill lamellae, separated for increased access, in $\mathrm{Mg}^{++}$and $\mathrm{Ca}^{++}$free ASW plus $2 \mathrm{mM}$ EDTA and $30 \mathrm{mM}$ Tris, $\mathrm{pH} 8.0$, for $1 \mathrm{hr}$, with intermittent agitation.

The resulting suspension of cells and cilia was strained through cotton gauze after the removal of the lamellar structures. This filtration removed large debris and mucus. The suspension was then centrifuged for 15 minutes at $400 \times g$ to pellet the cell bodies. Cilia were recovered from the supernatant by ultracentrifugation at $10,000 \times g$ for $15 \mathrm{~min}$ and were resuspended in complete ASW to give a concentration of $10^{6} \mathrm{cilia} / \mathrm{ml}$.

\section{CILIARY AGGLUTINATION ASSAY}

Serial doubling dilutions of the cilia suspension of equal volume $(0.1 \mathrm{ml})$ were added to the same $(0.1 \mathrm{ml})$ volume of the adult or fetal sera being tested. Parallel tests were made using the cilia suspension as the constant concentration and varying the dilution of sera with ASW. The mixtures were incubated at room temperature for $1 \mathrm{hr}$ and examined.

In a further set of experiments, serial doubling dilutions of isolated cilia or whole adult sera (volume, $0.025 \mathrm{ml}$ ) were incubated with $0.05 \mathrm{ml}$ of undiluted serum or cilia suspension, respectively, and $0.025 \mathrm{ml}$ of either $50 \mathrm{mM}$ dithiothreitol (final concentration, $12.5 \mathrm{mM}$ ) or $200 \mathrm{mM}$ 2-mercaptoethanol (final concentration, $50 \mathrm{mM}$ ) buffered to $\mathrm{pH} 8.0$ with Tris at a final concentration of $0.5 \mathrm{M}$.

\section{SERUM COLLECTION}

The following sera were used: human (adult and umbilical cord), adult bovine (ABS), fetal bovine (FBS), adult sheep, fetal sheep, rabbit, dog, and chicken.

In each case, the serum was separated from blood clotted at $4^{\circ} \mathrm{C}$ and stored in aliquots at $-20^{\circ} \mathrm{C}$. FBS was obtained from Flow Laboratories (Irvine, Scotland) whereas other animal blood was collected shortly after death. Normal human serum was obtained from adult volunteers and non-CF hospitalized children when possible; CF sera were obtained, with the informed consent of parents and doctors, from confirmed CF patients.

\section{SERUM DIALYSIS}

Serum dialysis is essential before its use in the Mytilus assay if serious osmotic damage to ciliated tissues is to be avoided. Dialysis against ASW using visking tubing $(6.3 \mathrm{~mm}$ diameter, nominal cut off at 3,000 daltons; Medicell International) was achieved overnight at $4^{\circ} \mathrm{C}$.

\section{$\gamma$ GLOBULIN FRACTION}

The $\gamma$ globulin fraction of various sera was obtained using an ammonium sulphate precipitation (38). Two precipitations using 45 and $40 \%\left(\mathrm{NH}_{4}\right)_{2} \mathrm{SO}_{4}$, followed by centrifugation and washing. isolated this fraction, which was then redissolved in ASW, and finally dialyzed to remove any $\left(\mathrm{NH}_{1}\right)_{2} \mathrm{SO}_{4}$.

\section{COMPLEMENT DESTRUCTION}

Where inactivation of the complement components of ABS was required, this was achieved by heating the serum to $56^{\circ} \mathrm{C}$ for 30 min in a water bath.

\section{PHOTOGRAPHY}

Ciliary activity (beating and metachronism) was recorded by high-speed cinephotography, slit camera photography, or a combination of stroboscopic illumination and still photography. Highspeed cinephotography was achieved using a Reichert microscope fitted with differential interference contrast optics, together with a Mitchell high-speed camera synchronized at 100 to 500 frames/ sec with a Chadwick Helmuth flash unit. The principle of the slit camera has been described by Takahashi and Murakami (52). The films used were Ilford Mark V, HP5, and FP4.

\section{SCANNING ELECTRON MICROSCOPY (SEM)}

Preparations for SEM were fixed either in $2 \% \mathrm{OsO}_{4}$ for $1 \mathrm{hr}$ followed by $4 \%$ glutaraldehyde overnight in the appropriate buffer solutions (in ASW buffered with $10 \mathrm{mM}$ Tris for Mytilus or in phosphate-buffered saline for rabbit material) or by the freeze substitution method of Barlow and Sleigh (3).

The specimens were dehydrated by a series of graded alcohols (ethanol), transferred to liquid $\mathrm{CO}_{2}$, critical point dried, mounted, and coated with gold-palladium. Examination took place in a Jeol JP-15 microscope.

\section{RESULTS}

A preliminary study showed that ciliary dyskinesia was produced inconsistently in Mytilus lateral cilia by human sera from both normal and CF donors. At a dilution of 50\% in ASW, some sera produced a strong disruption of activity, whereas others had little effect. Upon further dilution. the effect of each serum type was reduced (Table 1 ). The dyskinesia observed is clearly not restricted to $C F$ sera.

Experiments using $50 \% \mathrm{ABS}$ in ASW produced a very strong dyskinesia within 30 to $60 \mathrm{sec}$. This effect is reproducible irrespective of the individual variation of sera or sea mussels, differences only being noted in the exact time before the onset of dyskinesia. However, the time between application of a serum and dyskinesia rarely exceeded $3 \mathrm{~min}$.

The appearance of the normal wave pattern and the dyskinetic or altered wave form induced by ABS are shown by light microscopy and SEM in Figures 2 and 3, respectively. The conventional understanding of the meaning of dyskinesia (literally imperfect movement) has been interpreted as a "loss of synchrony": however, it is the metachrony, displayed in the wave form of active cilia that is disturbed. Cilia do not beat in true synchrony (i.e., with simultaneous effective strokes) but beat in metachronal sequences as portrayed in Figures 2 and 3. Direct observation of the dyskinetic activity without the aid of stroboscopic lighting gives the false impression of increased activity with a loss of metachrony. Examination with a stroboscope or of slit camera recordings (Fig. $2, c$ and $d$ ) clearly shows that a regular pattern still exists, although the form the new beat pattern differs greatly from the normal state. The metachrony of the cilia has changed in that the wavelength has increased up to seven times or more. Although there is an obvious change in wavelength, it is of interest to find that the beat frequency has remained steady (Fig. 4). These observations are clearly recorded in the slit camera traces (Fig. 2, $c$ and $d$ ).

The wavelength $(\lambda)$ of each metachronal wave is proportional to the number of repetitive sequences in the width of a trace:

$$
\begin{gathered}
\text { From Figure } 2 c \frac{\text { Normal wavelength }(\lambda \mathrm{N})}{\text { Abnormal wavelength }\left(\lambda_{\mathrm{A}}\right)}=\frac{6.5}{0.9}=7.2 \\
\text { From Fig. } 2 d \frac{\lambda \mathrm{N}}{\lambda_{\mathrm{A}}}=\frac{6.5}{2.5}=2.6
\end{gathered}
$$

Table 1. The effect of normal and $\mathrm{C} F$ sera from different

\begin{tabular}{|c|c|c|c|}
\hline \multicolumn{4}{|c|}{ Serum concentration } \\
\hline \multicolumn{2}{|c|}{$50 \%$} & \multicolumn{2}{|c|}{$1 \%$} \\
\hline $\begin{array}{c}\text { Dyskine- } \\
\text { sia' } \\
+ \text { ve }\end{array}$ & $\begin{array}{l}\text { Dyskine- } \\
\text { sia } \\
\text {-ve }\end{array}$ & $\begin{array}{c}\text { Dyskine- } \\
\text { sia }^{\prime} \\
+\mathrm{ve}\end{array}$ & $\begin{array}{c}\text { Dyskine- } \\
\text { sia' } \\
\text {-ve }\end{array}$ \\
\hline 6 & 2 & 2 & 4 \\
\hline 8 & 2 & 1 & 5 \\
\hline
\end{tabular}
individuals on the lateral cilia of Mytilus edulis

$\begin{array}{lllll}\text { CF serum } & 6 & 2 & 2 & 4 \\ \text { Normal serum } & 8 & 2 & 1 & 5\end{array}$

'Negative results are those in which no dyskinesia was seen within a 30-min observation period. 

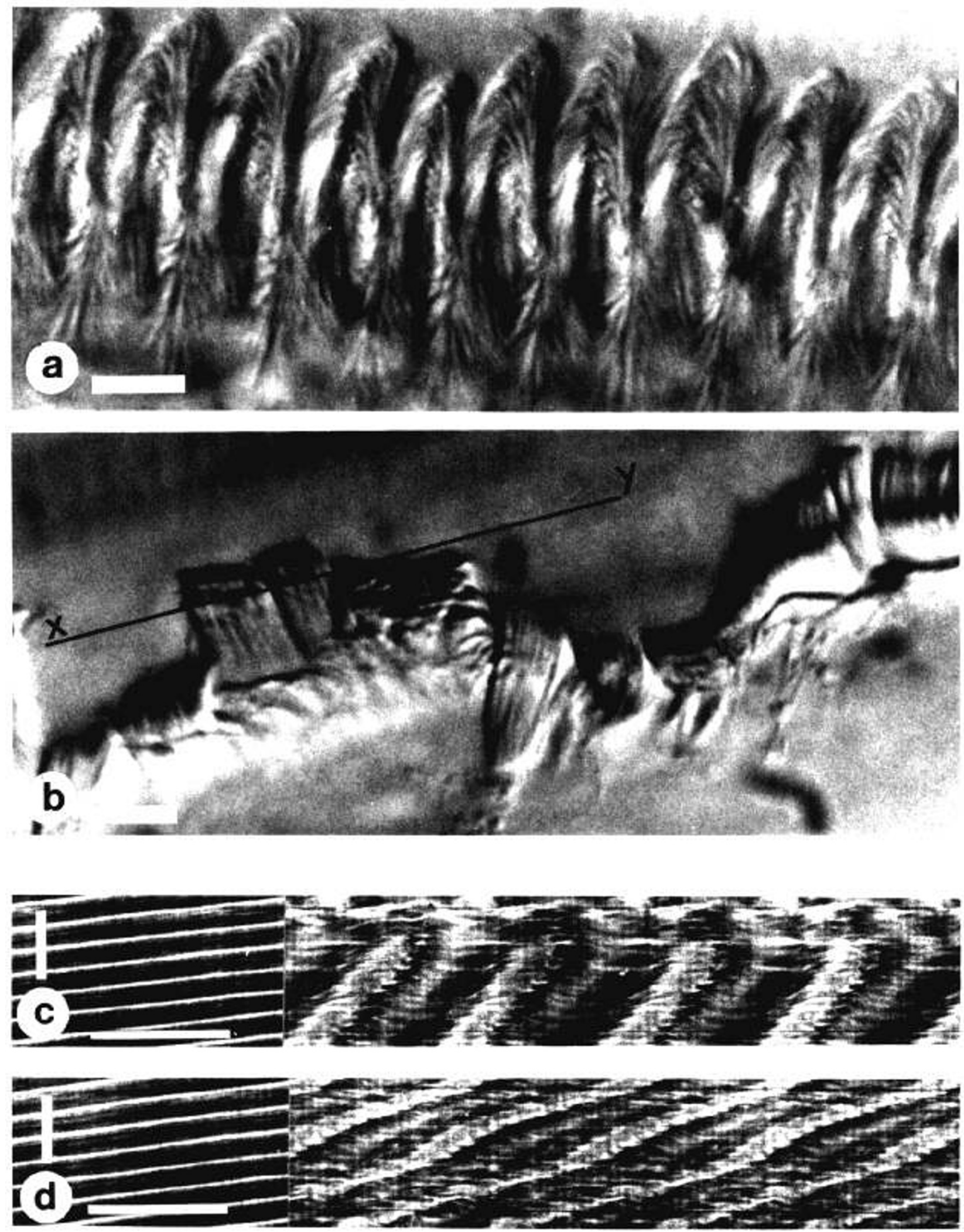

Fig. 2. a, the normal metachronal wave pattern of actively beating lateral cilia of the sea mussel Mytilus edulis, stimulated by $10 \div \mathrm{M} 5-\mathrm{HT}$ in ASW. The effective stroke is directed towards the base of the micrograph. and the metachronal wave travels from right to left. Magnification bar $=10 \mu \mathrm{m}$.

$b$, an abnormal or dyskinetic wave pattern of the lateral cilia of Mytilus induced by $50 \%$ ABS in ASW with $10^{5} \mathrm{M}$ 5-HT. The effective stroke is directed towards the base of the micrograph and the metachronal wave travels from right to left. Magnification bar $=10 \mu \mathrm{m}$.

Individual cilia are clearly visible in $a$ but the cilia in $b$ are agglutinated into blocks. The distance $\mathrm{x}-\mathrm{y}$ represents a single wavelength of the abnormal wave, which is equivalent to seven normal wavelengths.

$c$ and $d$, two slit camera recordings are shown with the normal wave pattern to the left and the abnormal or dyskinetic wave pattern to the right. $c$. dyskinetic wave with a wavelength over seven times greater than the normal: $d$, increased wavelength of approximately three times. Magnification bar: vertical $=30 \mu \mathrm{m}$; horizontal bar, representing time, $=50 \mathrm{msec}$ 

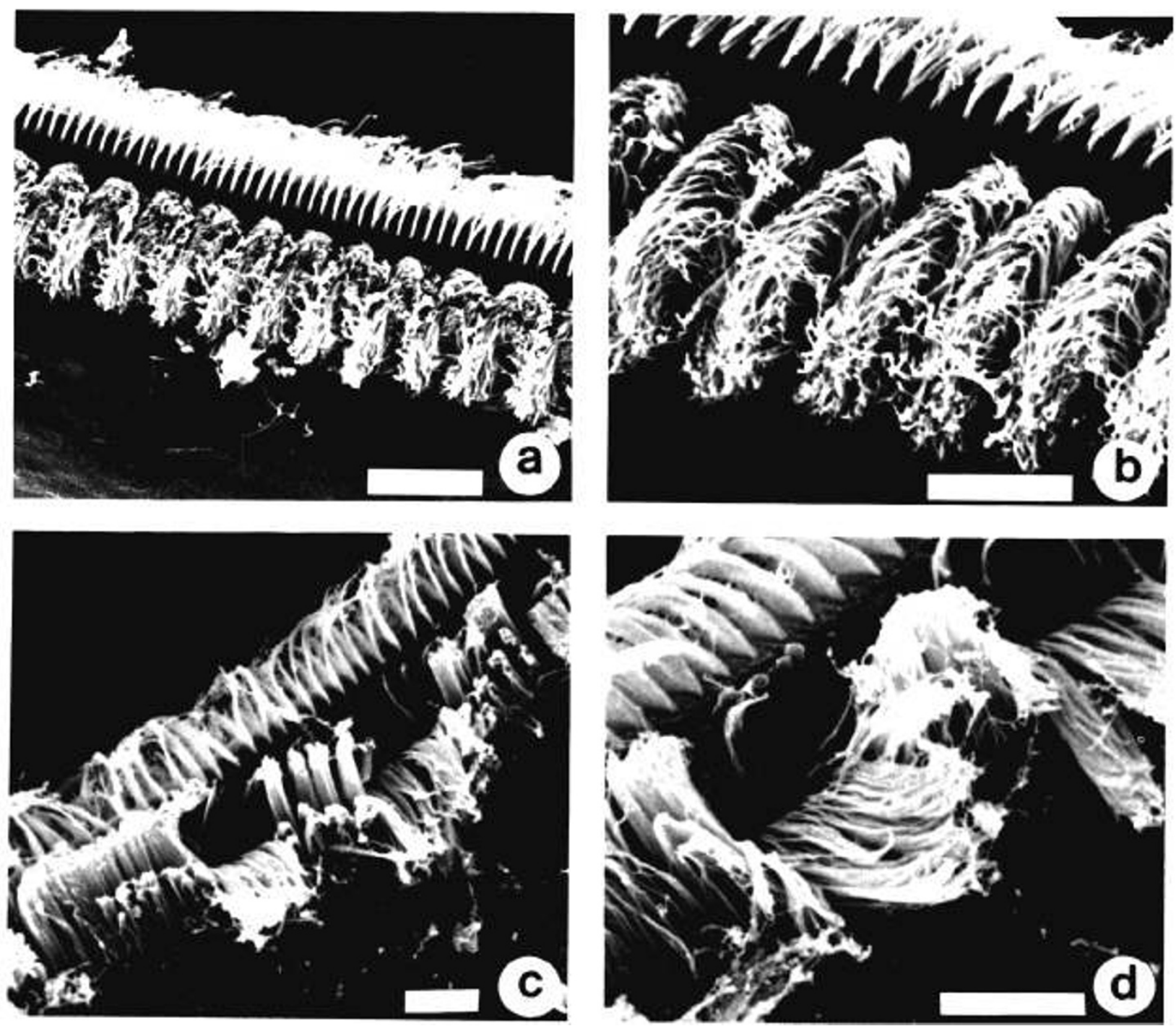

Fig. 3. SEMs of the normal wave pattern of actively beating lateral cilia of Mytilus edulis: at $(a)$ low power, $($ magnification bar $=20 \mu m)$; and $(b)$ higher power, (magnification bar $=10 \mu \mathrm{m}$ ). The waves have been stimulated by 10 " $\mathrm{M} 5-\mathrm{HT}$ and form a regular pattern, the effective stroke beating towards the base of the micrograph with the metachronal wave travelling from right to left. Individual cilia at the various stages of the beat cycle are clearly visible.

The dyskinetic wave $(c)$ at low power, (magnification bar $=10 \mu \mathrm{m})$ and $(d)$ higher power $($ magnification bar $=10 \mu m)$, clearly shows the cilia agglutinated into blocks which form the lengthened wave. The effective stroke is directed towards the bottom of the picture.

This increase in wavelength is apparent in the light micrographs (Fig. $2 b$ ): the distance marked $\mathrm{x}-\mathrm{y}$, being equivalent to $\lambda \mathrm{A}$. corresponds approximately to seven normal wavelengths (Fig. 2a).

Beat frequency (f) is inversely proportional to the distance (D) between two repetitive sequences along the horizontal axis.

From Fig. 2c $\frac{\text { Normal frequency }\left(\mathrm{f}_{\mathrm{N}}\right)}{\text { Abnormal frequency }\left(\mathrm{f}_{\mathrm{A}}\right)}=\frac{\mathrm{DN}_{\mathrm{N}}}{\mathrm{DA}_{\mathrm{A}}}=\frac{31}{28}-1$ From Fig. $2 d \frac{f_{N}}{f_{A}}=\frac{D_{N}}{D_{A}}=\frac{28}{29} \bumpeq 1$

There is clearly very little change in frequency in either example. although wavelength increases markedly.

The wave velocity is related to wavelength and frequency in the expression

$$
\text { Wave velocity }=\lambda \mathbf{f}
$$

The substantial increase in wave velocity of cilia treated with ABS gives an appearance of increased activity of the dyskinetic cilia although the frequency of beat has not changed.
A second characteristic of the abnormal wave is the appearance of ciliary blocks within the undulating ribbon of cilia, each block beating in an almost planar manner with a reduction in the lateral swing that is normally a prominent feature of the recovery stroke. The formation of these blocks does not appear to be completely random, each block being similar in size and the blocks being arranged in rows along the filament (Fig. 3). Often each unit corresponds to the position of an underlying ciliated cell. Within this arrangement, a weak antiplectic coordination is visible, the most ventral ciliary blocks beating slightly earlier than the more dorsal blocks (Fig. 3c). The main line of metachrony is directed laeoplectically as in the normal wave situation. It is, however, not uncommon to observe metachronal waves travelling in the opposite direction over short distances; some areas have metachronal waves moving out in both directions from an area of strongly agglutinated cilia.

After the discovery of the strong dyskinetic response induced by bovine sera, a number of other sera were examined to determine the extent of this phenomenon. Table 2 lists those sera and serum proteins tested and their effect on the Mytilus lateral cilia.

The responses shown to the positive sera were very similar to the effects caused by bovine sera: the cilia continued to beat with 


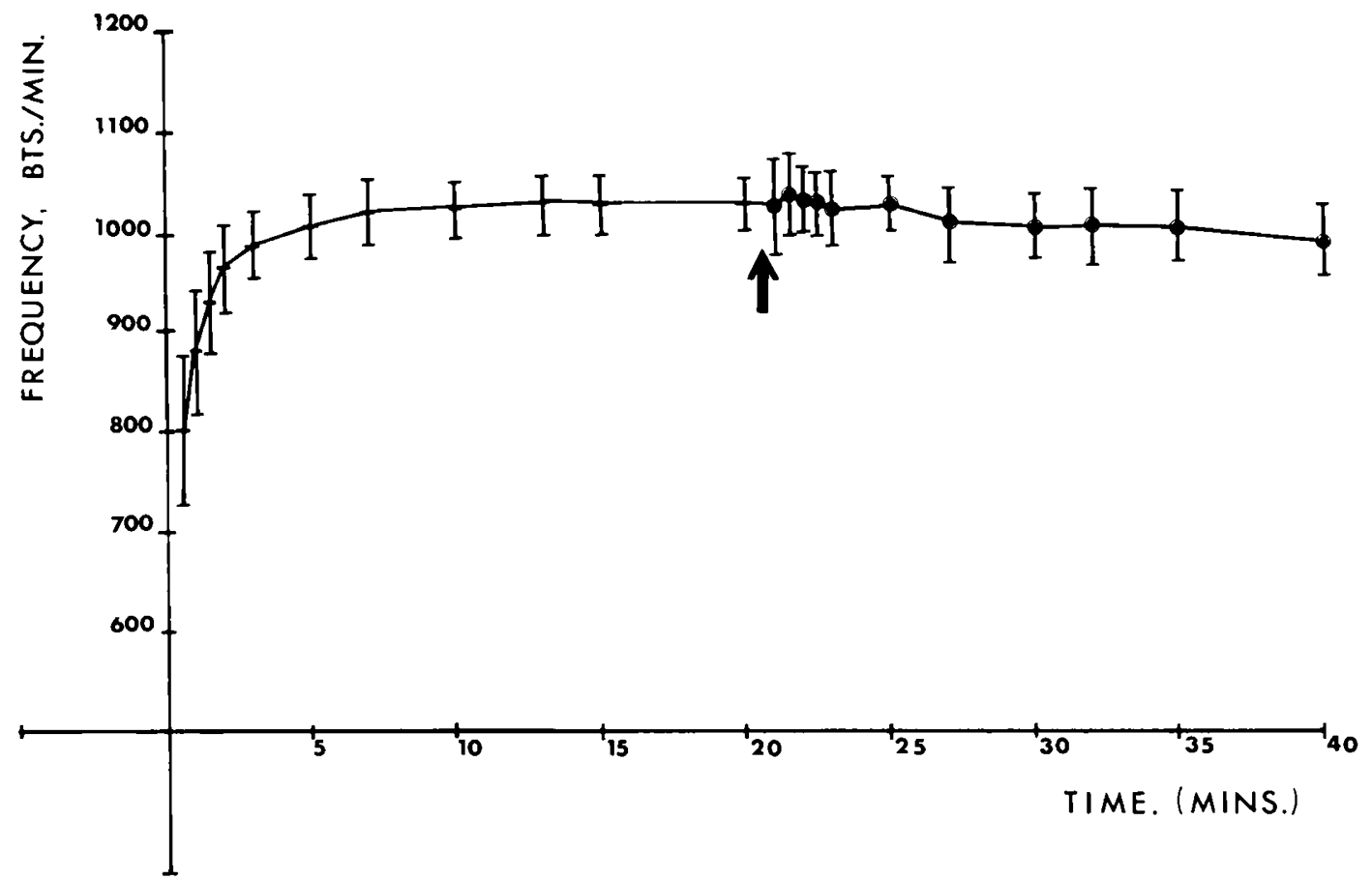

Fig. 4. A graph showing the effect of $10^{\circ} \mathrm{M} 5-\mathrm{HT}$ in ASW followed by the perfusion (arrow') of 50\% ABS in ASW + 10 ${ }^{5} \mathrm{M} 5-\mathrm{HT}$ (๑) on the beat frequency of the lateral cilia of Mytilus edulis with respect to time. The wave form represented by was completely dyskinetic due to the ABS. Points, mean of eight observations; vertical bars, S.E.

the same frequency, but the cilia agglutinated into blocks and the metachronal wavelength increased to an extent that varied in different sera. The metachronal coordination of the abnormal wave varied with the sample; some gill filaments showed extensive repetitive waveforms whereas others showed different rhythms in different parts, consistent patterns extending over two to three abnormal wavelengths. Slight variation was noted in the various sera for the time before the onset of dyskinesia.

Negative results were obtained with fetal bovine and fetal sheep sera and with human umbilical cord serum. Dyskinesia did not occur even when $100 \%$ sera of these types were used in place of the normal $50 \% ;$ in each case, the metachronal waves continued normally for well over an hour.

The $\gamma$ globulin fraction of bovine serum, prepared by ammonium sulphate precipitation, produced ciliary dyskinesia like that produced by whole sera. whereas bovine albumin had no effect. Deactivation of the complement components of bovine serum by heating did not influence the form of dyskinesia produced.

In those cases where sera produced ciliary dyskinesia, the removal of the sera by washing with several changes of ASW and 5-HT rarely restored the wave pattern to normal.

\section{RABBIT ASSAY}

Parallel studies using the rabbit assay were completed so that comparisons between this work and previous studies would be possible. It was found that $100 \%$ adult bovine sera produced dyskinesia in rabbit tracheal cilia after approximately $4 \mathrm{~min}$ Previous work with human sera, either healthy or CF, did not produce such clearly convincing results: in $100 \%$ serum, dyskinesia did not begin until 12 to $20 \mathrm{~min}$ had elapsed: over $30 \mathrm{~min}$ were required for onset of dyskinesia in 50\% human serum.

Upon the addition of ABS, as with human sera, there is an initial appearance of increased activity followed by the formation of clumps or groups of cilia which beat with a "nodding" type of action. This clumping effect is clearly observed by SEM (compare Fig. $5 b$ with $5 a$ ). The effect of bovine sera on rabbit cilia appears to be very similar to that on Mytilus cilia; the cilia in both cases are clearly agglutinated to form clumps.

The effect of diluting the serum was to increase the time before
Table 2. The effects of various animal sera and serum proteins on the lateral cilia of Mytilus edulis

\begin{tabular}{|c|c|c|}
\hline Sera & $\%$ & Dyskinesia' \\
\hline \multicolumn{3}{|l|}{ Adult } \\
\hline Sheep & 50 & + ve \\
\hline Dog & 50 & +ve \\
\hline Rabbit & 50 & + ve \\
\hline Fetal Bovine & 100 & - ve \\
\hline Fetal Sheep & 100 & $-v e$ \\
\hline Human Cord & 100 & $-v e$ \\
\hline Heated ABS & 50 & +ve \\
\hline Bovine $\gamma$ globulin ${ }^{2}$ & & +ve \\
\hline Bovine albumin ${ }^{2}$ & & $-v e$ \\
\hline
\end{tabular}

${ }^{1}$ Negative results are those in which no dyskinesia was seen within a 60-min period of observation.

" Bovine albumin and bovine $\gamma$ globulin used at a concentration equivalent to that in $50 \%$ ABS.

dyskinesia could be clearly distinguished (Fig. 6). For bovine sera on rabbit cilia, this period dramatically increases when serum concentrations become low, small differences in concentration giving rise to large delays before the onset of visible dyskinesia. The response of rabbit cilia to human sera is already comparatively slow at $100 \%$, becoming greatly retarded at even modest dilutions.

In comparison with the rabbit assay, the Mytilus assay shows dyskinesia in response to ABS in less than 5 min at concentrations as low as $5 \%$. The lag phase before dyskinesia then increases substantially with lowered concentration as found with the rabbit assay.

\section{ISOLATED CILIA}

The effect of sera on cilia was studied by a second method using isolated Mvtilus gill cilia. Treatment with adult sera (bovine or sheep) or the $\gamma$ globulin fraction of bovine sera brought about the agglutination of individual cilia in suspension. Fetal or cord sera had no such effect (Fig. 5, $c$ and $d$ ). This agglutination of the cilia 

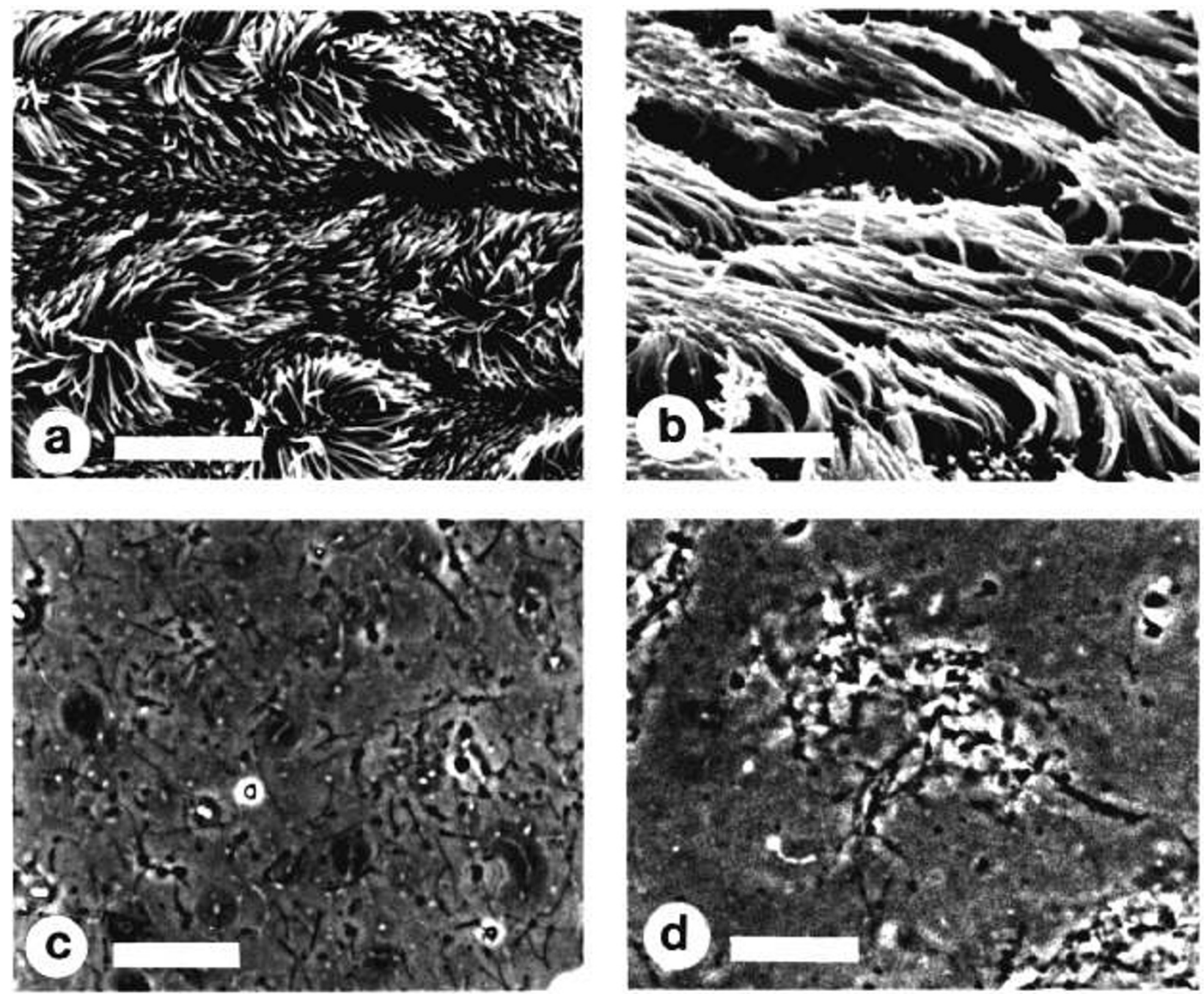

Fig. 5. SEMs showing $(a)$ the normal appearance of cultured rabbit tracheal cilia, each individual cilium being clearly separate and areas of activity being visible (magnification bar $=10 \mu \mathrm{m}$ ): and $(b)$ the effect of $\mathrm{ABS}$ on the rabbit tracheal cilia, where the cilia have become clumped together (magnification bar $=5 \mu \mathrm{m}$ ). $c$ shows a suspension of isolated gill cilia from Mitilus, (magnification har $=20 \mu \mathrm{m}$ ), which become agglutinated $(d)$ by ABS, (magnification bar $=20 \mu \mathrm{m}$ ).

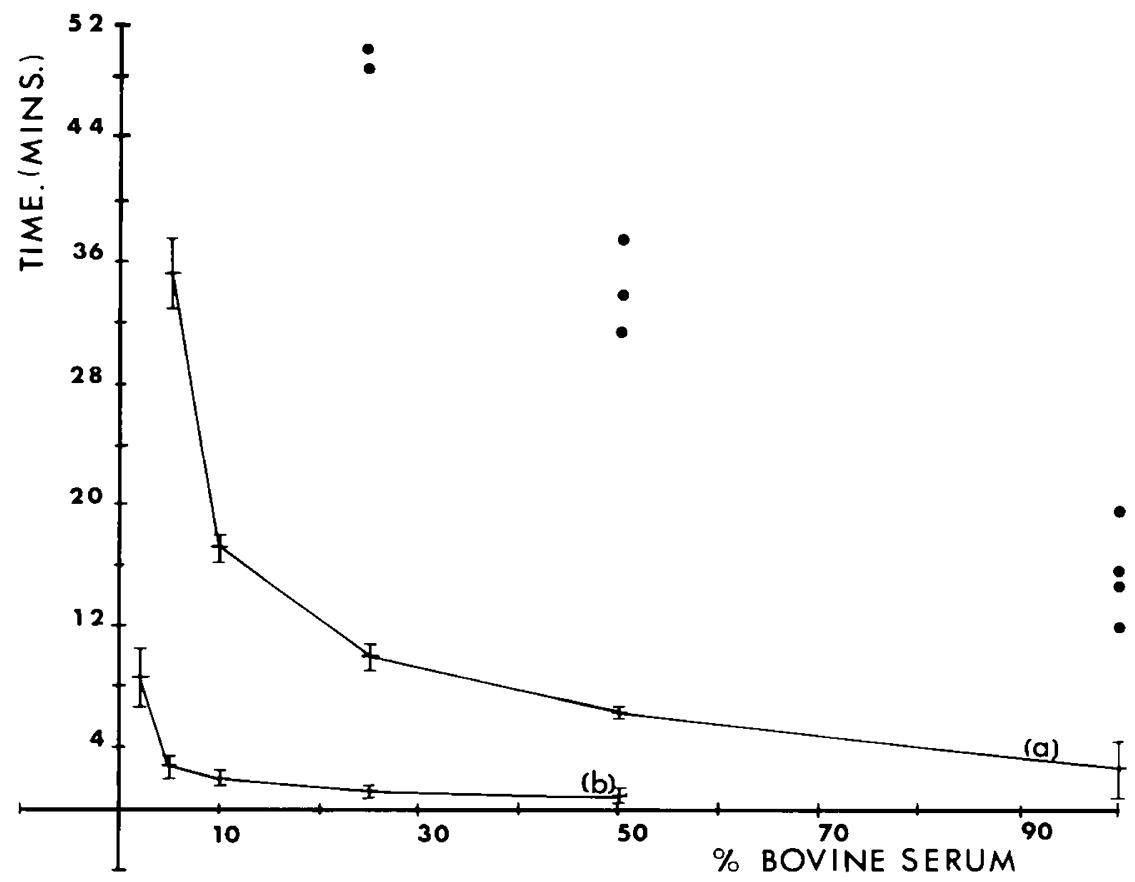

Fig. 6. A graph showing the effect of the dilution of ABS on the time taken before dyskinesia is tirst observed using (a) the rabbit tracheal cilia assay, or (b) the Mytilus lateral cilia assay. 0 . individual trials with rabbit cilia assay using various concentrations of human serum. 
by adult sera was prevented if $12.5 \mathrm{mM}$ dithiothreitol or $50 \mathrm{mM}$ mercaptoethanol was present in the experimental mixture. Either of these agents dissociates $19 \mathrm{~S}$ immunoglobulin $\mathrm{M}$ (IgM) to $8 \mathrm{~S}$ $\operatorname{IgM}(46)$.

\section{DISCUSSION}

Dialyzed sera from a variety of animals disrupts the normal ciliary activity of the lateral cilia of Mytilus; several of these sera also disrupt the activity of rabbit tracheal cilia. It was not possible to detect a consistent differential response between normal human sera and CF sera using either assay. There have been reports that sera from other non-CF pulmonary diseases, e.g., bronchial asthma, produce ciliary dyskinesia (23). Our evidence supports the contention that ciliary dyskinesia is not diagnostic of cystic fibrosis but is a phenomenon induced by many sera types.

Studies with Mytilus allow a more precise description of the nature of the serum-induced dyskinesia which has facilitated the understanding of how this new pattern may occur. The appearance and wavelength of the metachronal wave of the lateral cilia of Mytilus or any other field of cilia is related to the interactions between actively beating cilia. During each beat cycle, a cilium will influence its neighboring cilia by forces transmitted through the intervening fluid. This hydrodynamic coupling causes the adjacent cilia to beat slightly out of phase with each other so as to minimize interference; the ciliated field is then said to possess metachrony. The extent to which each cilium affects the surrounding cilia will depend on the degree of hydrodynamic coupling.

The normal wave pattern of Mytilus lateral cilia has a wavelength of about 10 to $12 \mu \mathrm{m}$ with a relatively low coupling force between individual cilia. In the dyskinetic wave form, the cilia were observed to aggregate together so that the unit of activity was enlarged from a single cilium to a cluster or block of many cilia. This agglutination has the effect of greatly increasing the coupling between individual cilia within ciliary blocks, which results in the increased wavelength observed in the dyskinetic wave. A second consequence of the ciliary agglutination is the absence of the lateral swing of the cilia during their recovery stroke: because of this change, the definite polarity of metachronism would no longer be firmly imposed on the cilia, explaining the observation of waves of coordination spreading in both directions from a patch of strongly agglutinated cilia. An interesting feature of the dyskinetic wave is the stability of the beat frequency; an increase in wave velocity results from the change in wavelength, giving the impression of increased activity.

This explanation of the cause of the change in ciliary activity is supported by the constancy of the beat frequency. Any change in the internal physiology of the ciliated cells due to serum factors would be expected to modify beat frequency, just as the frequency of Mytilus frontal gill cilia is affected by changes in concentration of $\mathrm{K}^{+}$or $\mathrm{Mg}^{++}$ions (36) or the frequency of the lateral cilia of Mytilus gill is affected by 5 -HT concentration $(1,34)$. Changes in the calcium concentration within ciliated cells, whether caused by external ionic changes $(47,53,55)$ or by the administration of calcium ionophore A23187 $(45,55)$ are normally accompanied by changes in beat frequency of the cilia. The effects on cilia of Mytilus when treated with sera, as reported here, are quite different from the effects of A23187 as reported by Murakami and Takahashi (45). This conflicts with the report by Bogart et al. $(11,12)$ that A23187 mimics the dyskinesia of rabbit cilia induced by sera.

Although we are confident that ciliary agglutination occurs when adult mammal sera are added to rabbit tracheal cilia, the change in activity cannot be described with such precision as in the Mytilus assay.

The absence of dyskinesia when Mytilus cilia are treated with fetal or cord sera implicates immunoglobulins as the cause of agglutination. The fetal sera used contain no immunoglobulins, and human cord serum contains only IgG. It appears then that either IgM or IgA may be responsible for ciliary agglutination. This suggestion is supported by the fact that isolated cilia are aggregated by sera and serum $\gamma$ globulin fractions which cause dyskinesia. Further, the treatment of sera with reducing agents (dithiothreitol and mercaptoethanol), which dissociate the sulphide bridges that maintain the polymer structures of immunoglobulins, destroyed the ability of the sera to agglutinate cilia. The $19 \mathrm{~S}$ pentamer of $\operatorname{IgM}$ is reduced to an $8 \mathrm{~S}$ monomer which has a reduced ability to cross link binding sites. $\operatorname{IgA}$ molecules are known to be dimeric in secretions and could have agglutination properties, but the $\operatorname{IgA}$ in sera are mainly monomeric and should not be altered by mild reduction. It is tentatively concluded that the agent in certain sera that causes the agglutination of cilia, and hence ciliary dyskinesia, is $\operatorname{IgM}$. This hypothesis is the subject of a subsequent study (50).

Other workers have found that the ciliary dyskinesia factor active in their assays was not an immunoglobulin $(28,37)$, although it could be found in association with $\operatorname{lgG}$ subclass I (14, 28 ). According to these studies, the factor has a molecular weight of 5,000 to 10,000 daltons $(5,14,20)$ : pentameric IgM has a molecular weight of 990,000 daltons (46). The small factor has been found in cell culture media $(9,13,27)$, saliva $(32)$, and in urine (15); preliminary tests using urine on the Mytilus assay showed no change in the ciliary activity, but cell culture fluids or saliva have not been examined. One suggested role for the factor is as a component of the complement system, particularly C 3 ( 22 . $24,25)$; other workers contest this suggestion (40). If sera are heated to destroy complement. then the ability to cause dyskinesia of Mytilus cilia is unimpaired. A small molecular weight factor should have been retained during dialysis, yet many $\mathrm{CF}$ sera showed negligible dyskinesia with the Mytilus assay whereas some normal sera similarly treated produced dyskinesia on filaments from the same mussel.

We are unable to account for these different results of different ciliary assays. The diverse tissues could be a possible source of variation, but the principal results to be compared have been obtained with marine oysters and mussels, which are similar. especially in their cilia. Indeed, the membranes of cilia of oysters and humans have common antigens, as Herzberg et al. (37) found when antisera produced in rabbits in response to oyster cilia cross reacted with human tracheal cilia.

One important difference between the Mytilus assay and any of its predecessors used to detect dyskinesia caused by sera is the precise quantitative data obtainable. In no previous case has a clear description of dyskinesia been given. A description of the character of the dyskinesia allows an analysis of the cause of the dyskinesia and the identification of the factor responsible. Baur et al. (7) completed a similar ultramicroscopic study of the effects of sera fractions on the oyster gill assay; mucus plugs were observed to become entangled with or flatten the cilia, but no clear description of the dyskinesia was given. Many of the cilia in their micrographs of gills treated with either normal or CF sera fractions were severely clumped or matted together. The seawater control, the only micrograph at low power, clearly showed a uniform covering of individual separate cilia. The only quantitative feature available in most other assays has been the time of exposure to serum before dyskinesia could be seen; we have shown that small changes in the concentration of bovine serum can cause large changes in the time taken to induce dyskinesia. Previous assays were therefore presumably directed towards detecting differences in concentration of a serum factor. If they were detecting differences in the concentration of ciliary-specific $\lg M$ between normal and $C F$ sera, then there could be some common explanation for the different findings; however, the Mytilus assay did not show consistent time differences between the onset of dyskinesia of normal or CF sera. The concentrations of immunoglobulins in normal sera is not significantly different from CF sera, but this does not exclude the possibility that individual variation may exist with respect to ciliary-specific IgM.

A vital precaution in studies of this type, particularly when using marine molluscs, is to match the osmolarity of the test sample with seawater. Failure to do so results in severe osmotic damage consisting of cell loss, ciliary swelling, especially at the tips, mucus production, and the accumulation of debris at the 
ciliated border. It is not always clear that this precaution has been appreciated, especially because some descriptions of dyskinesia include observations that can not be distinguished from normal osmotic damage.

It is interesting to speculate why serum $\gamma$ globulins should attach to cilia and cause agglutination. It could be that the presence of ciliated or flagellated protozoa in the gut of mammals (particularly cows and sheep) have stimulated the formation of ciliary antibodies. Alternatively, ciliary membranes may carry surface antigens identical or similar to those found on other infectious organisms to which the mammals are exposed. A wellknown example of this form of natural immunity is the blood group antigens, IgM being responsible for the agglutination of the foreign blood. An alternative assay that has been used to detect the CF serum factor depends on the agglutination of the bacterium Proteus by sera (19).

\section{CONCLUSION}

Sera from a variety of animals have been shown to cause an alteration of the metachronal coordination of the lateral cilia of the gill from the sea mussel Mytilus edulis. Cilia displaying this dyskinetic pattern have a longer metachronal wave, although the beat frequency remains stable; the wave velocity is consequently increased and, without the aid of stroboscopic lighting, one gains an impression of an increased and uncoordinated activity.

The lengthening of the dyskinetic wave is the result of an increased coupling between the cilia brought about by the agglutation of adjacent cilia into blocks by the serum immunoglobulins, IgM being strongly implicated. Ionic imbalances are not suspected because the beat frequency remains stable.

Our results are at variance with many other workers using ciliary assays because the ciliary dyskinesia is not restricted to $\mathrm{CF}$ or even to human sera, the responsible factor being a large immunoglobulin (perhaps IgM, 990,000 daltons) and not a small protein (10,000 daltons). A differential response between $C F$ and normal sera was not detected.

Although we are unable to explain these differences, the Mytilus assay has the major advantage of providing a precise description of ciliary dyskinesia, a characteristic absent from all other assays. The serum-induced dyskinesia observed in the Mytilus is not specific to $C F$, although concentrations of ciliary-specific or crossreacting immunoglobulins may be elevated in CF patients; small differences in serum concentrations greatly lengthen the time taken before the onset of dyskinesia.

\section{REFERENCES AND NOTES}

1. Aiello, E.: The control of ciliary activity in metazoa. In: M. A. Sleigh: Cilia and Flagella. p. 353-376 (Academic Press, Inc., London, 1974).

2. Aiello, E., and Sleigh, M. A.: The metachronal wave of lateral cilia of Mytilus edulis. J. Cell Biol., 54: 493 (1972).

3. Barlow, D. I., and Sleigh, M. A.: Freeze substitution for preservation of ciliated surfaces for scanning electron microscopy. J. Microsc. (Oxf.), 115: 81 (1979).

4. Barnett, D. R., Kurosky, A., Bowman, B. H., and Barranco, S. C.: Loss of the ciliary inhibitor effect of the cystic fibrosis factor following proteolytic digestion and heat denaturation. Tex. Rep. Biol. Med., 31: 697 (1973).

5. Barnett, D. R., Kurosky, A., Bowman, B. H., Hutchison. H. T., Schmoyer, I., and Carson, S. D.: Cystic fibrosis: molecular weight estimation of the ciliary inhibitor. Tex. Rep. Biol. Med., 31: 703 (1973).

6. Barnett, D. R., Schanfield, M. S., Mccombs, M. L., and Bowman, B. H.: Isoelectric focussing and $\mathrm{IgG}$ allotyping of the serum fraction containing $\mathrm{CF}$ ciliary inhibitor. Tex. Rep. Biol. Med., 31: 709 (1973).

7. Baur, P. S., Brinkley, B. R., and Bowman, B. H.: Effect of CF serum ciliary inhibitor on oyster gill ultrastructure: analysis by scanning and transmission electron microscopy. Tex. Rep. Biol. Med., 34: 155 (1976).

8. Bayne, B. L.: Marine Mussels; Their Ecology and Physiology. (University Press, Cambridge, 1976).

9. Beratis, N. G., Conover, J. H., Conod, E. J., Bonforte, R. J., and Hirschhorn, K.: Studies on ciliary dyskinesia factor in cystic fibrosis. III. Skin fibroblasts and cultured amniotic cells. Pediatr. Res., 7: 958 (1973).

10. Besley, G. T. N., Patrick, A. D., and Norman, A. P.: Inhibition of the motility of gill cilia of Dreissensia by plasma of cystic fibrosis patients and their parents. J. Med. Genet., 6: 278 (1969).

11. Bogart, B. I., Conod, E. J., and Conover, J. H.: The biologic activities of cystic fibrosis serum. I. The effects of cystic fibrosis sera and calcium ionophore A23187 on rabbit tracheal explants. Pediatr. Res., II: 131 (1977).
12. Bogart. B. I., Conod, E. J., Gaerlan, P. F., and Conover, J.: The biologic activities of cystic fibrosis serum. II. Ultrastructural aspects of the effects of cystic fibrosis sera and calcium ionophore A23187 on rabbit tracheal explants. Pediatr. Res. 12: 15 (1978)

13. Bowman, B. H., Barnett, D. R., Matalon, R., Danes, B. S., and Bearn, A. G Cystic fibrosis: fractionation of fibroblast media demonstrating ciliary inhibition. Proc. Natl. Acad. Sci. U. S. A.. 70: 548 (1973).

14. Bowman, B. H., Lankford, B. J., Fuller, G. M., Carson, S. D., Kurosky, A., and Barnett, D. R.: The ciliary inhibitor is a small polypeptide associated with immunoglobulin G. Biochem. Biophys. Res. Commun., 64: 1310 (1975)

15. Bowman, B. H., Lankford, B. J., McNeely, M. C., Carson, S. D., Barnett, D. R. and Berg, K.: Cystic fibrosis: studies with the oyster ciliary assay. Clin. Genet. 12: 333 (1977).

16. Bowman, B. H., Lockhart, L. M., and McCombs, M. L.: Oyster ciliary inhibition by cystic fibrosis factor. Science (Wash. D. C.), 164: 325 (1969).

17. Cherry, J. D., Roden, V. J., Rejent, A. J., and Dorner, R. W.: The inhibition of cilia activity in tracheal organ cultures by sera from children cystic fibrosis and control subjects. J. Pediatr., 79: 937 (1971).

18. Christensen, J., and Norrevang. A.: Studies on the effects of CF-serum on fresh tissue from Dreissenia gills, oyster gills and rabbit tracheal mucosa. Fifth International Cystic Fibrosis Conference, Churchill College, Cambridge. Paper No. 6, p. 49 (1969).

19. Cohen, F. L., and Daniel, W. L.: Effect of cystic fibrosis sera on Proteus vulgaris motility. J. Med. Genet., 11: 253 (1974)

20. Conod, E. J., Conover, J. H., and Gaerlan, P.: Separation of serum ciliary dyskinesia substance from cystic fibrosis subjects. Pediatr. Res., 11: 45 (1977)

21. Conover, J. H. Bonforte, R. J., Hathaway, P. Paciuc, S., Conod, E. J., Hirschhorn, F. B., and Kopel, F. B.: Studies on ciliary dyskinesia factor in cystic fibrosis. I. Bioassay and heterozygote detection in serum. Pediatr. Res., 7: 220 (1973).

22. Conover, J. H., and Conod, E. J.: Complement in cystic fibrosis. Lancet, 1: 47 (1975).

23. Conover, J. H.. Conod, E. J., and Hirschhorn, K.: Ciliary dyskinesia factor in immunological and pulmonary disease. Lancet, $I$ : 1194 (1973).

24. Conover, J. H., Conod. E. J., and Hirschhorn, K.: Complement components in cystic fibrosis. Lancet, 2: 1501 (1973).

25. Conover, J. H., Conod, E. J., and Hirschhorn, K.: Studies on ciliary dyskinesia factor in cystic fibrosis. IV. Its possible identification as an anaphylatoxin (C3a)-IgG complex. Life Sci., 14: 253 (1974).

26. Crawford, M. d'A.: Experience of the cilia test for a factor in the serum of patients and carriers of cystic fibrosis. Fifth International Cystic Fibrosis Conference, Churchill College, Cambridge. Paper No. 5. p. 42 (1969).

27. Danes, B. S., and Bearn. A. G.: Oyster ciliary inhibition by cystic fibrosis culture media. J. Exp. Med.. 136: 1313 (1972).

28. Danes, B. S., Litwin, S. D., Hutteroth, T. H., Cleve, H., and Bearn. A. G. Characterization of cystic fibrosis factor and its interaction with human immunoglobulin. J. Exp. Med., 137: 1538 (1973).

29. Di Sant'Agnese, P. A., and Davis, P. B.: Research in cystic fibrosis (First of 3 parts). N. Engl. J. Med., 295: 481 (1976).

30. Di Sant'Agnese, P. A., and Davis, P. B.: Research into cystic fibrosis (Second of 3 parts). N. Engl. J. Med., 295: 534 (1976).

31. Di Sant'Agnese, P. A., and Davis, P. B.: Research into cystic fibrosis (Third of 3 parts). N. Engl. J. Med., 295: 597 (1976).

32. Doggett, R. G., and Harrison, G. M.: Cystic fibrosis: reversal of ciliary inhibition in serum and saliva by heparin. Tex. Rep. Biol. Med., 31: 685 (1973).

33. Gibbons, I. R.: Chemical dissection of cilia. Arch. Biol. Liège, 76: 317 (1965).

34. Gosselin, R. E.: The cilioexcitatory activity of Serotonin. J. Cell Comp. Physiol., 58: 17 (1961).

35. Gosselin, R. E., Moore, K. E., and Milton, A. S.: Physiological control of molluscan gill cilia by 5-hydroxytryptamine. J. Gen. Physiol., 46: 277 (1962).

36. Gray, J.: Ciliary Movement. p. 95 (University Press, Cambridge, 1928).

37. Herzberg. V., Calvert, L., and Bowman, B. H.: Evidence that the ciliary inhibitor of cystic fibrosis is not an antibody. J. Clin. Invest., 52: 2732 (1973).

38. Hudson, L., and Hay, F. C.: Practical Immunology. (Blackwell Scientific Publications, Oxford, 1976).

39. Jørgensen, C. B.: On gill function in the mussel Mytilus edulis. Ophelia, 13: 187 (1975).

40. Lieberman, J.: Complement components in cystic fibrosis. Lancet, $1: 1230$ (1974)

41. Linck, R. W.: Comparative isolation of cilia and flagella from the lamellibranch mollusc Aequipecten irradians. J. Cell Sci., 12: 345 (1973).

42. Lockhart, L. H., and Bowman, B. H.: Assays for the detection of the cystic fibrosis gene. Tex. Rep. Biol. Med., 31: 631 (1973).

43. McCombs, M. L.: Research in cystic fibrosis: a review. Tex. Rep. Biol. Med., 31 615 (1973).

44. McCombs, M. L., and Bowman, B. H.: Rivanol treatment of cystic fibrosis serum: effect of supernatant upon ciliary action. Clin. Genet., 1 : 171 (1970).

45. Murakami, A., and Takahashi, K.: The role of calcium in the control of ciliary movement in Mytilus. II. The effects of calcium ionophores X537A and A23187 on the lateral gill cilia. J. Fac. Sci. Univ. Tokyo, 13: 251 (1975).

46. Nisonoff, J. E., Hooper, J. E., and Spring, S. B.: The antibody molecule. p. 86 (Academic Press, Inc., New York, 1975).

47. Paparo, A., and Murphy, J. A.: The effect of $\mathrm{Ca}^{++}$on the rate of beating of the lateral cilia in Mytilus edulis. I. Response to perfusion with SHT, DA, BOL and PBZ. Comp. Biochem. Physiol. C Comp. Pharmacol., 50: 9 (1975).

48. Posselt, H. G., and Bender, S.: Heterozygote testing in cystic fibrosis. Z. Kinderheilkd., 110: 93 (1971).

49. Sanderson, M. J. and Sleigh, M. A.: The beat pattern of rabbit tracheal cilia. J. Cell Sci. (in press). 
50. Sanderson. M. J.. and Sleigh. M. A.: The agglutination of cilia by serum immunoglobulins. (in preparation).

51. Spock. A. Heick. H. M. C.. Cress, H., and Logan. W. S.: Abnormal serum facto in patients with cystic fibrosis of the pancreas. Pediatr. Res., 1: 173 (1967).

52. Takahashi, K.. and Murakami. A.: Nervous inhibition of ciliary motion in the gill of the sea mussel Myrilus edulis. J. Fac. Sci. Univ. Tokyo, 11: 359 (1968).

53. Tsuchiya, $\mathrm{T}$ : $\mathrm{Ca}^{++}$-induced arrest response in Triton-extracted lateral cilia of Mvtilus gill. Experientia, 32: 1439 (1976).

54. Verdugo, P.. Hinds. T. R.. and Vincenzi. F. F.: Laser light scattering spectroscopy: preliminary results on bioassay of cystic fibrosis factors. Pediatr. Res.. 13: 131 (1979).

55. Walter, M. F.. and Satir, P.: Calcium control of ciliary arrest in mussel gill cells J. Cell Biol., 74: $110\left(\begin{array}{ll}1 & 8\end{array}\right)$.

56. Wood, R. E., Boat. T. F.. and Doershuk, C. F.: State of ant: cystic fibrosis. Am.
Rev. Respir. Dis.. 113: 833 (1976).

57. The authors would like to thank David Barlow for his expertise with the preparation of specimens for SEM by freeze substitution and useful discussion, Chris Hawkins for his assistance with SEM. and Helen Linford and Christine Matthews for their help with the text and figures.

58. Requests for reprints should be addressed to: Dr. M. J. Sanderson. Department of Anatomy. Albert Einstein College of Medicine, 1300 Morris Park Ave. Bronx. NY 10461 (USA).

59. This research was supported initially by the Cystic Fibrosis Research Trust and completed with support from Southampton University Committee for Advanced Studies.

60. Received for publication January $28,1980$.

61. Accepted for publication July 15.1980. 